\title{
One Case of a BRCA1 Germ Line Mutation Ovarian Carcinoma Patient Based on Abnormal Immunohistochemistry Finding
}

\author{
Hyun Joo Kim · Jung Min Park • Hyoun Wook Lee ${ }^{1}$ Eun Hee Lee ${ }^{1}$ Min Kyu Kim² \\ Department of Obstetrics and Gynecology, Samsung Medical Center, Seoul; 'Department of Pathology, ${ }^{2}$ Division of Gynecologic Oncology, Department of Obstetrics and \\ Gynecology, Samsung Changwon Hospital, Sungkyunkwan University School of Medicine, Changwon, Korea
}

Ovarian cancer is one of the most lethal gynecological cancers in the Western world. In 2012, approximately 2,000 new patients and 980 mortalities due to ovarian cancer were reported in Korea. Due to the lack of efficient diagnostic methods for early detection and rapid progression to advanced stages, patients with ovarian cancer have poor survival rates. As hereditary ovarian cancers tend to present as higher grade disease in younger patients, screening for hereditary ovarian cancer is important for decreasing prevalence and improving patient survival.

$B R C A 1$ and $B R C A 2$ are mismatch repair genes accounting for $85 \%$ of hereditary breast and epithelial ovarian cancers. ${ }^{1}$ They are located on chromosomes 17q21 (22 exons, 80-kb DNA) and 13q12-13 (26 exons, 70-kb DNA), ${ }^{2,3}$ respectively. Other mismatch repair genes also contribute to carcinogenesis. ${ }^{4}$ The average lifetime risk of developing breast and ovarian cancers in women with a BRCA1 mutation are $65 \%$ and $39 \%$, respectively, and the corresponding estimates for BRCA2 are $45 \%$ and $11 \%$, respectively. ${ }^{5}$ Three methods are commonly used to manage BRCA mutation carriers: screening, prophylactic surgery, and chemoprevention. Salpingo-oophorectomy in carriers of $B R C A$ mutations can decrease the risk of breast cancer and $\mathrm{BR}$ CA-related gynecologic cancer. ${ }^{6}$

We report a case of germ line BRCA1 mutation in an ovarian cancer patient without a family history of breast cancer detected

\section{Corresponding Author}

Min Kyu Kim, M.D.

Division of Gynecologic Oncology, Department of Obstetrics and Gynecology, Samsung Changwon Hospital, Sungkyunkwan University School of Medicine, 158 Paryong-ro, Masanhoewon-gu, Changwon 630-723, Korea

Tel: +82-55-290-6040, Fax: +82-2-6442-9285, E-mail: minkyukim@skku.edu

Received: July 31, 2013 Revised: October 2, 2013

Accepted: October 17, 2013 with immunohistochemistry screening. We carried out gene sequencing for BRCA1 and BRCA2 after a constructive counseling with the patient and detected a deletion of ATTGGGCA at codon 1824 in exon 24 of the BRCA1 gene.

\section{CASE REPORT}

A 52-year-old woman complaining of constipation and abdominal distension was referred to our hospital from a local clinic. Computerized axial tomography scans showed a $12-\mathrm{cm}$ solid and cystic ovarian mass with a large amount of ascites and right pleural effusion (Fig. 1). Her cancer antigen-125 level was 432.4 $\mathrm{U} / \mathrm{mL}$. After other laboratory tests were performed, we conducted a staging exploratory laparotomy. Pathology showed grade 3, stage IV serous papillary adenocarcinoma from the ovary invading the pelvic cavity and right pleura. The patient underwent adjuvant taxane and carboplatin combination chemotherapy via six cycles of paclitaxel $\left(175 \mathrm{mg} / \mathrm{m}^{2}\right)$ plus carboplatin of the area under the curve of $5 \mathrm{mg}$ given on day one of a 21-day cycle.

The patient declined to discuss her family history and her family members were not contacted for further information. However, she approved genetic testing after counseling. Immunohistochemistry (IHC) screening of the mismatch repair genes BRCA1 and BRCA2 was performed, showing abnormal immunoreactivity for BRCA1 (negative staining) and focal positive reaction for BRCA2 (Fig. 2). Gene sequencing for BRCA1 and $B R C A 2$ was conducted after counseling and a deletion of ATTGGGCA at codon 1824 in exon 24 was detected, with the stop codon (TGA) appearing in 1826 [5470_5477del8 (p.Ile1824AspfsX3)]. This frame shift mutation produced a truncated protein (Fig. 1). 

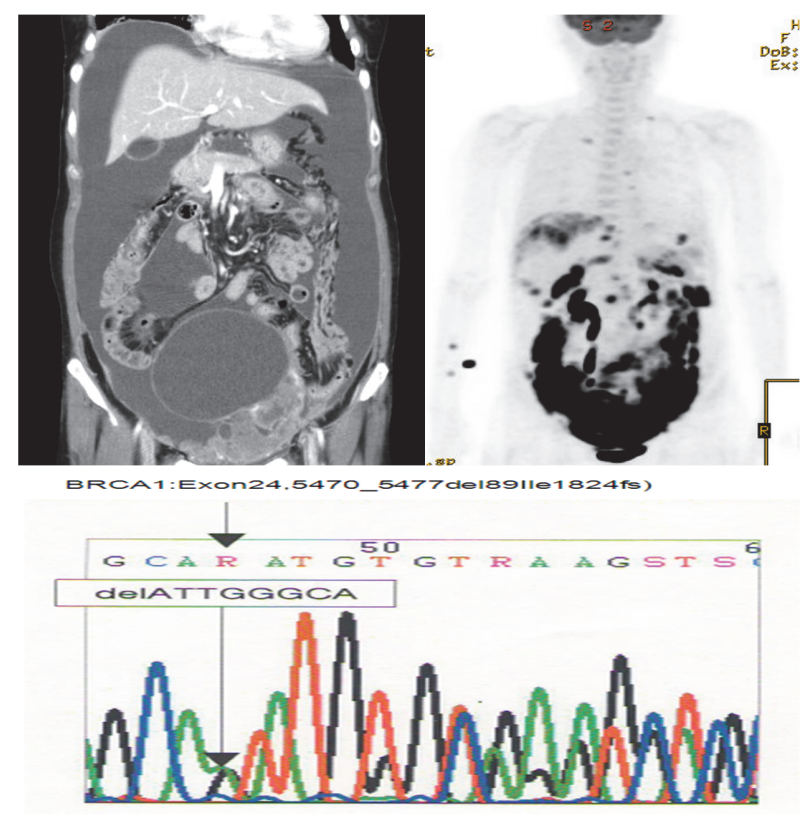

Fig. 1. Computerized axial tomography scan and gene sequencing results.

\section{DISCUSSION}

This case demonstrates that IHC screening can be helpful for detecting $B R C A$ mutations in sporadic ovarian cancer patients with unknown family histories. We retrospectively confirmed a germ line mutation after observing abnormal findings on IHC for BRCA. Unfortunately, we could not persuade the patient's family members to undergo genetic testing for the mutation.

The frequency of BRCA1 or BRCA2 mutations in general population is estimated to be 1 in 300 to 1 in $800 .^{7}$ There are many types of $B R C A$ mutations with differences in penetration. Such mutations have previously been reported in Korean breast cancer patients, but not in patients with ovarian carcinoma. ${ }^{8}$

Although progress in molecular diagnosis has assisted in cancer diagnosis, ${ }^{9}$ the use of vague guidelines for selecting patients for genetic testing, high cost, and lack of insurance coverage make it difficult to detect hereditary cancer patients.

After diagnosis, three methods are commonly applied to manage $B R C A$ mutation carriers: screening, prophylactic surgery, and chemoprevention. Our patient and her family were offered genetic counseling by gynecologic oncologist and breast cancer surgeon. Her breast mammogram and sonography showed negative results.

The average lifetime risk of developing breast and ovarian cancer in women with a BRCA1 mutation are $65 \%$ and $39 \%$, respectively. ${ }^{5}$ The penetrance of ovarian cancer in $B R C A 1 \mathrm{mu}-$
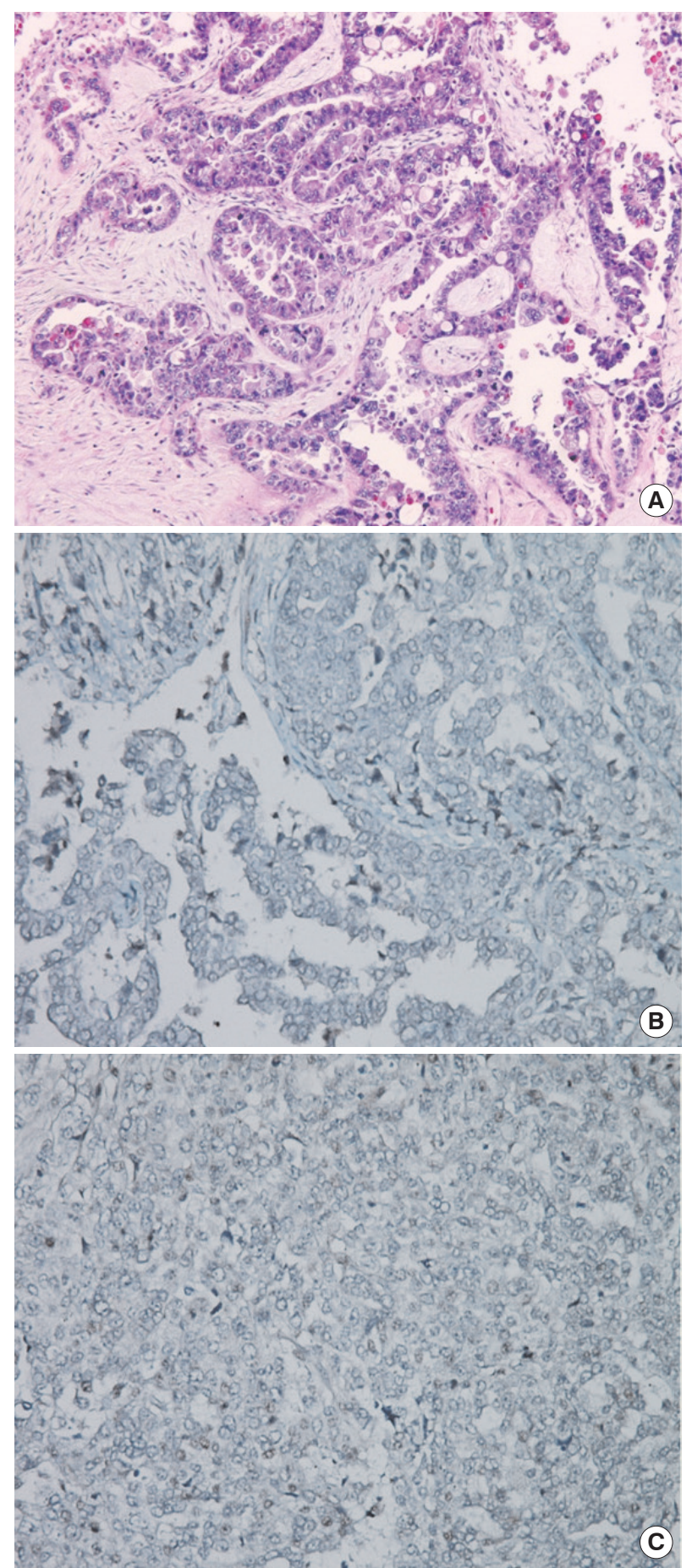

Fig. 2. (A) Hematoxylin and eosin staining of sample. (B) BRCA1 immunohistochemistry (IHC) (negative staining). (C) BRCA2 $\mathrm{HC}$ (focal positive staining). Agent: BRCA1 (Abcam ab16870), BRCA2 (Abcam ab110967). Evaluation: Nuclear staining of the tumor is scored as either 2 (strong, normal), 1 (weak), or 0 (negative, abnormal) compared with the corresponding internal control.

tation carriers is known to be $11 \%$ to $23 \% .^{10}$ The patient was offered counseling on breast cancer risks and prevention. 
As our abilities to recognize genetic risks improve, patients with an inherited risk of ovarian carcinoma can be identified before cancer onset, rendering effective prevention of ovarian carcinoma associated with inherited risks. IHC screening can help diagnose BRCA mutations, and clinicians should recommend genetic sequencing in patients with unknown family histories of disease.

\section{Conflicts of Interest}

No potential conflict of interest relevant to this article was reported.

\section{REFERENCES}

1. Lynch HT, Casey MJ, Snyder CL, et al. Hereditary ovarian carcinoma: heterogeneity, molecular genetics, pathology, and management. Mol Oncol 2009; 3: 97-137.

2. Miki Y, Swensen J, Shattuck-Eidens D, et al. A strong candidate for the breast and ovarian cancer susceptibility gene BRCA1. Science 1994; 266: 66-71.

3. Wooster R, Bignell G, Lancaster J, et al. Identification of the breast cancer susceptibility gene BRCA2. Nature 1995; 378: 789-92.
4. Kang YK, Kim WH. Microsatellite instability and mismatch repair protein (hMLH1, hMSH2) expression in intrahepatic cholangiocarcinoma. Korean J Pathol 2005; 39: 9-14.

5. Antoniou A, Pharoah PD, Narod S, et al. Average risks of breast and ovarian cancer associated with BRCA1 or BRCA2 mutations detected in case series unselected for family history: a combined analysis of 22 studies. Am J Hum Genet 2003; 72: 1117-30.

6. Kauff ND, Satagopan JM, Robson ME, et al. Risk-reducing salpingo-oophorectomy in women with a BRCA1 or BRCA2 mutation. $\mathrm{N}$ Engl J Med 2002; 346: 1609-15.

7. Whittemore AS, Gong G, Itnyre J. Prevalence and contribution of $B R C A 1$ mutations in breast cancer and ovarian cancer: results from three U.S. population-based case-control studies of ovarian cancer. Am J Hum Genet 1997; 60: 496-504.

8. Kim H, Cho DY, Choi DH, et al. Characteristics and spectrum of $B R C A 1$ and $B R C A 2$ mutations in 3,922 Korean patients with breast and ovarian cancer. Breast Cancer Res Treat 2012; 134: 1315-26.

9. Hwang TS. Molecular biologic techniques in cytopathologic diagnosis. Korean J Pathol 2009; 43: 387-92.

10. Eisen A, Lubinski J, Klijn J, et al. Breast cancer risk following bilateral oophorectomy in BRCA1 and BRCA2 mutation carriers: an international case-control study. J Clin Oncol 2005; 23: 7491-6. 